\title{
Un agenda global de plus en plus chargé : environnement, climat, biodiversité...
}

Le premier numéro de NSS date, déjà, de 17 ans... Quoi de neuf en cette fin d'année 2010 ?

Sur le front de l'interdisciplinarité, de nombreuses publications se sont accumulées, sans que, soyons francs, la question soit posée dans des termes bien différents de ceux d'autrefois. Le saut essentiel dans la réflexion reste dû au défunt programme Environnement, vie et sociétés du CNRS et, depuis, nous gérons l'héritage. Les industriels considèrent que l'essentiel des innovations sont à présent des « innovations de systèmes » et non plus des innovations de produits. Peut-être le temps est-il venu d'envisager que l'interdisciplinarité soit moins importante que la conception de nouveaux systèmes de recherche, partant des questions, des objets, pour aller aux savoirs disciplinaires et non l'inverse?

Le «développement durable», point de départ de NSS, a viré à l'incantation, au moulin à prières, et il se vérifie qu'il est bien commode de parler de générations futures pour s'épargner d'affronter les iniquités et inégalités présentes dont on voudrait bien qu'elles soient moins... durables. Plus "scientifiquement», les définitions qui en sont données de-ci de-là réfèrent moins à une problématique qu'à des éléments : «le développement, c'est trois piliers » (sic !). Sous-jacente aux discours, la fiction d'un "équilibre » est toujours bien présente : présente dans les têtes à défaut de l'être dans le réel, fait de variabilité, d'instabilité, de changement.

Le climat tend à se gâter, dans les sciences comme dans l'atmosphère. $\mathrm{Si}$, dans cette dernière, la connaissance et l'interdisciplinarité ont grandement progressé, l'idée que l'on se fait de la science a, singulièrement, été marquée par la grande confusion entre controverse scientifique et stérile polémique reposant sur des certitudes et des listes d'arguments en lieu et place de réfutation des hypothèses et des méthodes. Cette indigne polémique se déroulant sur un plan médiatique des plus sommaire, son enjeu était bien plus la prise en otage de l'opinion publique que l'avancée des questions et des connaissances. Par-delà la querelle avec les «climatosceptiques »- comme si le climat était affaire de foi -, la question qui reste ouverte est celle des relations entre science, médias, politiques. Cette question mériterait de donner lieu à colloques et numéros spéciaux de NSS, tant elle pèsera sur les années à venir.

Dans le champ de l'environnement, pourtant, le climat continue, 17 ans après, à tenir un rôle de « sujet » - la biodiversité étant envisagée comme contingente, objet subissant les conséquences du changement climatique. Cette vision des choses oublie que les humains font partie de la biodiversité qu'ils bouleversent et que cette évolution de la biodiversité fait le changement climatique, qui à son tour rétroagit sur sa cause première.

Pourtant, la surprise est venue de la biodiversité, du bilan d'une année 2010, « année mondiale de la biodiversité ». Au terme de celle-ci, il est possible de se réjouir de ce bilan, au rebours de points de vue chagrins entendus ici ou là : l'ambiance est à la catastrophe annoncée et au complot sur l'air de « on ne nous dit pas tout !»

Ce bilan peut s'exprimer en termes de décisions internationales, d'abord. Les réunions qui ont préparé la conférence des parties à la Convention sur la diversité biologique ont fait du bon travail. Le "programme de travail», qui porte sur les engagements concrets en moyens, a été financé ; le protocole sur « l'accès et le partage des avantages », relatif à la reconnaissance des droits des pays d'origine et des populations locales, approuvé. Le programme relatif aux "objectifs du millénaire » en matière de biodiversité a reçu des moyens et une confirmation de son importance dans le domaine de la lutte contre la pauvreté.

En ce qui concerne la communauté scientifique, la décision majeure de l'année, discutée à Busan, décidée à Nagoya et entérinée par l'Assemblée générale des Nations unies en décembre, réside dans la création d'un 
Groupe intergouvernemental sur la biodiversité et les services écosystémiques - ou, en anglais, IPBES (Intergovernmental Panel on Biodiversity and Ecological Services) -, souvent appelé, improprement, le « GIEC de la biodiversité ». La création de l'IPBES (www.ipbes.net) aura pris six ans : pour un accord entre tant de pays, il s'agit d'un délai très court. Puisse la communauté scientifique française prendre au sérieux la naissance de cet IPBES et $s^{\prime} y$ investir avec autant d'énergie que celle qu'elle déploie au sein du GIEC : l'année 2011 sera cruciale pour cela.

L'année qui vient pourrait être mise à profit par l'association NSS-Dialogues et par la revue pour analyser en profondeur les leçons à tirer des querelles médiatiques au regard des relations entre natures, sciences et sociétés. Le climat nous a livré d'abondants matériaux sur le thème. L'année internationale de la forêt ne manquera pas de nous en fournir d'aussi abondants : se dessine déjà, à travers les reportages diffusés par les télévisions, une autre belle querelle entre les tenants de la forêt comme écosystème et ceux de la forêt comme production de bois, comme champ d'arbres. Belle matière première en perspective pour NSS, et pour les forestiers qui y participent ! L'évolution des entreprises au regard du climat et de la biodiversité mériterait également plus d'attention, tant elle est rapide, aussi bien dans les grandes que dans les moins grandes. La conduite d'un groupe de travail au sein de l'association Orée (www.oree.org) en a fait une belle démonstration.

Les perspectives ne s'arrêtent pas à 2011. En 2012 se tiendra le Sommet de Rio, dit « Rio + 20 ». Il se dit qu'une Organisation mondiale de l'environnement pourrait y voir le jour, regroupant plusieurs agences actuelles des Nations unies : FAO, UNEP, PNUD ; il se dit qu'elle regrouperait aussi les trois grandes conventions : Climat, Biodiversité, Désertification. Il se dit enfin que les conditions sont à présent réunies pour que Rio +20 débouche sur un accord général sur le climat, de grande ampleur et contraignant. Il se dit beaucoup, mais une chose est sûre : ce sommet sera véritablement très important.

Les conférences de Nagoya et de Cancun on montré que de nombreux pays, développés, "émergents ", en développement, pouvaient s'entendre sur des enjeux remettant en cause leurs propres positions. Bonne nouvelle, car les habitants de la Terre vont devoir faire des choix cruciaux et parfois coûteux pour assurer un avenir vivable.

Ah! j'oubliais : il devrait aussi y avoir, en 2012, une élection d'importance locale, dans un pays d'Europe qui accepte difficilement que le monde ne tourne pas autour de lui... Espérons toutefois que le vol d'un papillon sur Paris puisse générer du beau temps sur Rio de Janeiro!

Jacques Weber 